\title{
Optimal Sequences and Sum Capacity of Symbol Asynchronous CDMA Systems
}

\author{
Jie Luo, Member, IEEE, Sennur Ulukus, Member, IEEE, and Anthony Ephremides, Fellow, IEEE
}

\begin{abstract}
The optimal signature sequences that maximize the sum capacity of a direct sequence code-division multiple-access (CDMA) system are characterized in the general case of symbol delay profile and user power constraints. It is shown that the optimal sum capacity of the symbol asynchronous system equals that of the symbol synchronous system with the same user power constraints. With the optimal signature sequence set, the maximum sum capacity is achieved with white Gaussian input signals. The existence of the optimal signature sequence set is proved by the proposal of an explicit construction method for arbitrary user delay profiles and power constraints.
\end{abstract}

Index Terms-Asynchronous code-division multiple access (CDMA), CDMA, optimal signature sequences, sum capacity.

\section{INTRODUCTION}

$\mathbf{I}_{\mathrm{N}}^{\mathrm{N}}$ $\mathrm{N}$ direct-sequence code-division multiple-access (DSCDMA) communications, users share the entire bandwidth with each other. The symbol waveform of a user signal is generated by spreading the chip waveform with its signature sequence or code. Due to the capability of offering high capacity, flexibility, and security, DS-CDMA systems became popular in the early 1980s and have been studied extensively since then [1]. Among the research works on CDMA, there has been special interest in understanding the impact of signature sequences on the sum capacity of the system. Suppose that $K$ and $N$ are the number of users and the spreading gain of the system, respectively. For the symbol synchronous CDMA system, when the user powers are equal, [2] showed that the sum capacity is maximized by assigning an orthogonal signature sequence set to the users when $K \leq N$, and by assigning a Welch-bound-equality (WBE) signature sequence set to the users when $K>N$. The general case of asymmetric user powers was solved in [3], where it was shown that the sum capacity is maximized by assigning orthogonal signatures to the oversized users (see (21) for a definition), and assigning a

Manuscript received May 24, 2004; revised April 19, 2005. This work was supported by the National Aeronautics and Space Administration Award NCC8-235, the Collaborative Technology Alliance for Communication and Networks sponsored by the U.S. Army Laboratory under Cooperative Agreement DAAD19-01-2-0011, and the National Science Foundation under Grant ANI-0205330. Any opinions findings, and conclusions or recommendations expressed in this material are those of the authors and do not necessarily reflect the views of the National Aeronautics and Space Administration or the Army Research Laboratory of the U.S. Government. The material in this paper was presented in part at the IEEE Global Telecommunications Conference, Dallas, TX, November 2004.

The authors are with the Electrical and Computer Engineering Department, University of Maryland, College Park, MD 20742 USA (e-mail: rockey@eng. umd.edu; ulukus@eng.umd.edu; tony@eng.umd.edu).

Communicated by R. R. Müller, Associate Editor for Communications.

Digital Object Identifier 10.1109/TIT.2005.851749 generalized WBE (GWBE) sequence set to the nonoversized users. A recursive construction [3] and an iterative method [4] were proposed to obtain the optimal sequence sets. The results have also been extended to the colored Gaussian noise in [5].

The signature sequence design problem for symbol asynchronous (but chip synchronous) systems was studied recently in [6] under the assumptions of fixed signal delay profile and frame synchronism. For a special case when the users are equal powered and when the matched filter receivers are used, the optimal sequence set that maximizes the user capacity has been found by minimizing the total squared asynchronous correlation (TSAC). An extension to the asymmetric user powers case was presented in [7], [8]. It has been shown that the constraints on the optimal signature sequences in a symbol asynchronous system are stricter compared to those in a symbol synchronous system. We refer to the optimal signature sequence set obtained in [6] (that achieves the TSAC lower bound) as the asynchronous WBE (AWBE) sequence set in this paper. Although simulations show that the AWBE sequences can be obtained by minimizing the TSAC, the existence of the AWBE sequence set under an arbitrary user delay profile was left as an open problem in [6], [8]. The combined power control and signature design problem when users have random delay profile (as opposed to a fixed delay profile as in [6], [8]) was studied in [9]. The common feature of the works presented in [6]-[9] is that the signature sequence design problem is studied with the criterion of maximum user capacity, and under the assumption of linear receivers.

In this paper, we study the signature sequence design problem from the sum information capacity point of view for asymmetric (i.e., unequal) user powers in a symbol asynchronous (but chip synchronous) CDMA system. We assume that the users are frame synchronous, and have a fixed deterministic delay profile. Under the assumption that the signature sequences are real-valued, we show that the sum capacity of the symbol asynchronous system is upper-bounded by that of the symbol synchronous system with the same power constraints. We derive the optimal signature sequences for the asynchronous system that achieve the sum capacity upper bound. Similar to the result in [3], we show that the optimal signature sequences for the symbol asynchronous system is obtained by assigning orthogonal signatures to the oversized users and assigning a generalized AWBE (GAWBE) signature sequence set to the nonoversized users. In the asynchronous case, the symbol delays limit our freedom in designing the optimal signature sequence set. For a general delay profile, the orthogonal signature sequences assigned to the oversized users are usually restricted to have only one nonzero chip in each signature. 
We show that, when the users are equal powered, the AWBE signature sequences that achieve the TSAC lower bound given in [6, Theorem 3] are indeed optimal. In addition, we present an explicit method to construct the optimal signature sequence set, given an arbitrary signal delay profile and general user power constraints. This solves the existence problem left open in [6]; in fact, it solves the existence problem in a more general setting of arbitrary powers.

The rest of the paper is organized as follows. The system model is presented in Section II. The sum capacity of the symbol asynchronous system is analyzed in Section III, where an upper bound on the sum capacity is given. We derive the necessary condition on the optimal signature sequence set that helps the system to achieve the sum capacity upper bound in Section IV-A. The conditions are also shown to be sufficient if the input signals have white power spectra. In Section IV-B, we present an explicit method to construct the optimal signature sequence set for an arbitrary given user delay profile and power constraints. The paper concludes in Section V.

\section{SySTEM MODEL}

We consider a DS-CDMA system where all $K$ users transmit to a single receiver. We assume that the user signals are chip synchronous; the chip waveform is identical for all users and is designed such that it can pass the bandwidth-limited channel with negligible distortion. ${ }^{1}$ The processing gain, the dimensionality of the signal space, is $N$.

Define

$$
\boldsymbol{s}_{k}=\left[\begin{array}{llll}
s_{k 1} & s_{k 2} & \ldots & s_{k N}
\end{array}\right]^{T}
$$

as the signature sequence of user $k$. We assume that the elements of $\boldsymbol{s}_{k}$ are real-valued ${ }^{2}$ and the energy of the signature sequence is normalized to 1 , i.e., $\boldsymbol{s}_{k}^{T} \boldsymbol{s}_{k}=1, \forall k$. Suppose that the symbol delay of user $k$ is $c_{k}$ chips, where $c_{k}=0,1, \ldots, N-1$. We divide the signature sequence of user $k$ into two parts. The subvector $\tilde{\boldsymbol{s}}_{k}^{L}$ that contains the first $N-c_{k}$ chips is termed the left signature sequence, and the subvector $\tilde{\boldsymbol{s}}_{k}^{R}$ that contains the last $c_{k}$ chips is termed the right signature sequence, i.e.,

$$
\begin{aligned}
\tilde{\boldsymbol{s}}_{k}^{L} & =\left[s_{k 1}, s_{k 2}, \ldots, s_{k\left(N-c_{k}\right)}\right]^{T} \\
\tilde{\boldsymbol{s}}_{k}^{R} & =\left[s_{k\left(N-c_{k}+1\right)}, s_{k\left(N-c_{k}+2\right)}, \ldots, s_{k N}\right]^{T} .
\end{aligned}
$$

In addition, define an $N \times 1$ vector $\boldsymbol{s}_{k}^{L}$ by padding 0 's to $\tilde{\boldsymbol{s}}_{k}^{L}$ from the top and define an $N \times 1$ vector $\boldsymbol{s}_{k}^{R}$ by padding 0 's to $\tilde{\boldsymbol{s}}_{k}^{R}$ from the bottom

$$
\boldsymbol{s}_{k}^{L}=[\overbrace{0, \ldots, 0}^{c_{k} 0 \mathrm{~s}}, \tilde{\boldsymbol{s}}_{k}^{L^{T}}]^{T}, \quad \boldsymbol{s}_{k}^{R}=[\tilde{\boldsymbol{s}}_{k}^{R^{T}}, \overbrace{0, \ldots, 0}^{N-c_{k} 0 \mathrm{~s}}]^{T} .
$$

\footnotetext{
${ }^{1}$ Here chip synchrony is assumed in order to preserve the tractability of the problem. In a practical system, where the user signals are chip asynchronous, a combined optimization problem involving the design of the signature sequences and the chip waveform subject to a bandwidth constraint should be considered.

${ }^{2}$ As opposed to binary signature sequences [10] or sequences whose entries take values from a general finite-alphabet set. This real signature sequence assumption, combined with the chip synchrony assumption, imply that our results give insights into the performance limits of practical systems.
}

Consider the first $M$ symbol durations. The chip matched filter output of the receiver can be denoted by an $M N \times 1$ real-valued column vector $\boldsymbol{y}$, which satisfies the following system model:

$$
\boldsymbol{y}=\sum_{k=1}^{K} \boldsymbol{S}_{k} \boldsymbol{x}_{k}+\boldsymbol{n}
$$

Here $\boldsymbol{y}=\left[\boldsymbol{y}(1)^{T}, \boldsymbol{y}(2)^{T}, \ldots, \boldsymbol{y}(M)^{T}\right]^{T}$, where $\boldsymbol{y}(m)$ is the chip-matched filter output vector of the $m$ th symbol duration; $x_{k}=\left[\begin{array}{llll}x_{k}(1) & x_{k}(2) & \ldots & x_{k}(M)\end{array}\right]^{T}$ is the source symbol vector of user $k ; \boldsymbol{n}$ is the Gaussian noise with zero mean and covariance matrix $E\left[\boldsymbol{n} \boldsymbol{n}^{T}\right]=\sigma^{2} \boldsymbol{I}_{M N}, \boldsymbol{I}_{M N}$ being the $M N \times$ $M N$ identity matrix; and $\boldsymbol{S}_{k}$ is the signature matrix of user $k$, which is given by

$$
\boldsymbol{S}_{k}=\left[\begin{array}{cccc}
\boldsymbol{s}_{k}^{L} & \mathbf{0} & \ddots & \mathbf{0} \\
\boldsymbol{s}_{k}^{R} & \boldsymbol{s}_{k}^{L} & \mathbf{0} & \ddots \\
\mathbf{0} & \ddots & \ddots & \mathbf{0} \\
\ddots & \mathbf{0} & \boldsymbol{s}_{k}^{R} & \boldsymbol{s}_{k}^{L}
\end{array}\right]
$$

We assume that both the symbols and the signature sequences are real valued. The average power of the normalized source signal of the $k$ th user is restricted to

$$
\operatorname{tr}\left(E\left[\boldsymbol{x}_{k} \boldsymbol{x}_{k}^{T}\right]\right) \leq M P_{k}
$$

where $P_{k}$ is the average power per symbol of user $k$. Given $J$ as an arbitrary group of users, the mutual information $I\left(\boldsymbol{x}_{k \in J} ; \boldsymbol{y} \mid \boldsymbol{x}_{k \notin J}\right)$ is given by [11]

$$
I\left(\boldsymbol{x}_{k \in J} ; \boldsymbol{y} \mid \boldsymbol{x}_{k \notin J}\right) \leq \frac{1}{2} \log _{2}\left[\left|\boldsymbol{I}_{M N}+\sum_{k \in J} \frac{\boldsymbol{S}_{k} E\left[\boldsymbol{x}_{k} \boldsymbol{x}_{k}^{T}\right] \boldsymbol{S}_{k}^{T}}{\sigma^{2}}\right|\right]
$$

with equality if the signals of users $k \in J$ are Gaussian. Here $|\cdot|$ denotes the determinant of a square matrix.

Assume that the user delay profile is known to both the transmitters and the receiver. ${ }^{3}$ The capacity region of the system is given by the convex closure, over independent random vectors $x_{k}$ satisfying (5), of the union of the following heptagons:

$$
\begin{aligned}
\lim _{M \rightarrow \infty} \bigcap_{J \subset\{1,2, \ldots, K\}}\left\{\left(R_{1}, \ldots, R_{K}\right):\right. & \\
& \left.0 \leq \sum_{k \in J} R_{k} \leq \frac{1}{M} I\left(\boldsymbol{x}_{k \in J} ; \boldsymbol{y} \mid \boldsymbol{x}_{k \notin J}\right)\right\} .
\end{aligned}
$$

Suppose that the signature sequences are given. In the situation when user signals are symbol synchronous, it is shown in [12] that the rate constraints are maximized if the source signals have white power spectra, i.e., $E\left[\boldsymbol{x}_{k} \boldsymbol{x}_{k}^{T}\right]=P_{k} \boldsymbol{I}_{M}, \forall k$. However, in the symbol asynchronous case, in general, there is no unique power spectrum that can maximize the rate upper bounds in (7) simultaneously [12].

${ }^{3}$ Note that the optimal signature design problem that maximizes the sum capacity when the transmitters do not have the information about the delay profile remains open. 
In the literature, the sum capacity of the rate region, defined as $\max \left(\sum_{k=1}^{K} R_{k}\right)$, has been of particular interest since it is a single number that represents the overall capacity limit of the system. Combining (6) and (7), the sum capacity per symbol of the system satisfies

$$
\begin{aligned}
C_{\text {sum }} \leq & \lim _{M \rightarrow \infty} \underset{\operatorname{tr}\left(E\left[\boldsymbol{x}_{k} \boldsymbol{x}_{k}^{T}\right]\right) \leq M P_{k}}{\max } \\
& \left.\frac{1}{2 M} \log _{2}\left[\mid \boldsymbol{I}_{M N}+\sum_{k=1}^{K} \frac{\boldsymbol{S}_{k} E\left[\boldsymbol{x}_{k} \boldsymbol{x}_{k}^{T}\right] \boldsymbol{S}_{k}^{T}}{\sigma^{2}}\right\rceil\right]
\end{aligned}
$$

where equality holds when the input signals are stationary Gaussian.

\section{UPPER BOUND ON THE SUM CAPACITY}

Let $\left\{\boldsymbol{A}_{M}\right\}$ and $\left\{\boldsymbol{B}_{M}\right\}$ be two sequences of matrices with realvalued entries, where both $\boldsymbol{A}_{M}$ and $\boldsymbol{B}_{M}$ are of size $M \times M$. Define the operator norm, or the strong norm of $\boldsymbol{A}_{M}$ as [13]

$$
\left\|\boldsymbol{A}_{M}\right\|=\max _{\boldsymbol{x}}\left\{\frac{\boldsymbol{x}^{T} \boldsymbol{A}_{M}^{T} \boldsymbol{A}_{M} \boldsymbol{x}}{\boldsymbol{x}^{T} \boldsymbol{x}}\right\}^{\frac{1}{2}}
$$

$\boldsymbol{A}_{M}$ and $\boldsymbol{B}_{M}$ are asymptotically equivalent [13] if i) $\left\|\boldsymbol{A}_{M}\right\|$ and $\left\|\boldsymbol{B}_{M}\right\|$ are uniformly bounded, i.e., there exists a $U$, such that

$$
\left\|A_{M}\right\|,\left\|B_{M}\right\| \leq U<\infty
$$

and ii)

$$
\lim _{M \rightarrow \infty}\left\{\frac{1}{M} \operatorname{tr}\left[\left(\boldsymbol{A}_{M}-\boldsymbol{B}_{M}\right)^{T}\left(\boldsymbol{A}_{M}-\boldsymbol{B}_{M}\right)\right]\right\}^{\frac{1}{2}}=0
$$

Suppose that the average powers per symbol of the user signals are upper-bounded. The following theorem shows that the signature matrices and their corresponding block circulant versions are asymptotically equivalent.

Theorem 1: Suppose that the average power per symbol of the signal of user $k$ is upper-bounded, for all $k$. Define the block circulant signature matrix of user $k$ by

$$
\hat{\boldsymbol{S}}_{k}=\left[\begin{array}{ccccc}
\boldsymbol{s}_{k}^{L} & \mathbf{0} & \ddots & \mathbf{0} & \boldsymbol{s}_{k}^{R} \\
\boldsymbol{s}_{k}^{R} & \boldsymbol{s}_{k}^{L} & \mathbf{0} & \ddots & \mathbf{0} \\
\mathbf{0} & \ddots & \ddots & \ddots & \ddots \\
\ddots & \ddots & \ddots & \ddots & \mathbf{0} \\
\mathbf{0} & \ddots & \mathbf{0} & \boldsymbol{s}_{k}^{R} & \boldsymbol{s}_{k}^{L}
\end{array}\right] .
$$

Then for any set of users $J$, the two matrices

$$
\sum_{k \in J} \hat{\boldsymbol{S}}_{k} E\left[\boldsymbol{x}_{k} \boldsymbol{x}_{k}^{T}\right] \hat{\boldsymbol{S}}_{k}^{T} \quad \text { and } \quad \sum_{k \in J} \boldsymbol{S}_{k} E\left[\boldsymbol{x}_{k} \boldsymbol{x}_{k}^{T}\right] \boldsymbol{S}_{k}^{T}
$$

are asymptotically equivalent.

The proof of Theorem 1 is provided in Appendix A.
Consequently, according to Theorem 1 and [13, Theorem 2.3], we can replace the signature matrices by their circulant versions and rewrite (8) as

$$
\begin{aligned}
C_{\text {sum }} \leq & \lim _{M \rightarrow \infty} \underset{\max \left(E\left[\boldsymbol{x}_{k} \boldsymbol{x}_{k}^{T}\right]\right) \leq M P_{k}}{ } \\
& \left.\frac{1}{2 M} \log _{2}\left[\mid \boldsymbol{I}_{M N}+\sum_{k=1}^{K} \frac{\hat{\boldsymbol{S}}_{k} E\left[\boldsymbol{x}_{k} \boldsymbol{x}_{k}^{T}\right] \hat{\boldsymbol{S}}_{k}^{T}}{\sigma^{2}}\right\rceil\right] .
\end{aligned}
$$

Note that all circulant matrices of the same dimension have identical eigenvector set. Define the $M \times 1$ vector $\boldsymbol{q}_{m}$ and the $M \times M$ Fourier transform matrix $\boldsymbol{Q}_{M}$ as

$$
\begin{aligned}
\boldsymbol{q}_{m} & =\left[\begin{array}{llll}
1 & e^{j \frac{2 \pi(m-1)}{M}} & \ldots & e^{j \frac{2 \pi(m-1)(M-1)}{M}}
\end{array}\right]^{H} \\
\boldsymbol{Q}_{M} & =\frac{1}{\sqrt{M}}\left[\begin{array}{llll}
\boldsymbol{q}_{1} & \boldsymbol{q}_{2} & \ldots & \boldsymbol{q}_{M}
\end{array}\right]
\end{aligned}
$$

where the superscript $H$ denotes the conjugate transpose. Denote the $m$ th component of vector $\boldsymbol{q}_{n}$ by $q_{n m}$. According to [13] and [14], we can decompose the block circulant matrix $\hat{\boldsymbol{S}}_{k}$ as

$$
\hat{\boldsymbol{S}}_{k}=\left[\begin{array}{ccc}
q_{11} \boldsymbol{I}_{N} & \ddots & q_{M 1} \boldsymbol{I}_{N} \\
\ddots & \ddots & \ddots \\
q_{1 M} \boldsymbol{I}_{N} & \ddots & q_{M M} \boldsymbol{I}_{N}
\end{array}\right] \boldsymbol{\Phi}_{k}^{*} \boldsymbol{Q}_{M}^{H}
$$

where the superscript $*$ denotes the conjugate operation; $\boldsymbol{\Phi}_{k}$ is a block diagonal matrix, defined as

$$
\boldsymbol{\Phi}_{k}=\left[\begin{array}{cccc}
\boldsymbol{\phi}_{k 1} & \mathbf{0} & \ddots & \mathbf{0} \\
\mathbf{0} & \boldsymbol{\phi}_{k 2} & \ddots & \ddots \\
\ddots & \ddots & \ddots & \ddots \\
\mathbf{0} & \ddots & \mathbf{0} & \boldsymbol{\phi}_{k M}
\end{array}\right]
$$

and $\phi_{k m}$ in (17) is an $N \times 1$ column vector given by

$$
\phi_{k m}=\boldsymbol{s}_{k}^{L}+\boldsymbol{s}_{k}^{R} e^{-j \frac{2 \pi(m-1)}{M}} .
$$

Substituting (16) into (14), we get

$$
\begin{aligned}
\mid \boldsymbol{I}_{M N} & +\sum_{k=1}^{K} \frac{\hat{\boldsymbol{S}}_{k} E\left[\boldsymbol{x}_{k} \boldsymbol{x}_{k}^{T}\right] \hat{\boldsymbol{S}}_{k}^{T}}{\sigma^{2}} \mid \\
& =\left|\boldsymbol{I}_{M N}+\sum_{k=1}^{K} \frac{\boldsymbol{\Phi}_{k}^{*} \boldsymbol{Q}_{M}^{H} E\left[\boldsymbol{x}_{k} \boldsymbol{x}_{k}^{T}\right] \boldsymbol{Q}_{M} \boldsymbol{\Phi}_{k}^{T}}{\sigma^{2}}\right| \\
& \leq\left|\boldsymbol{I}_{M N}+\sum_{k=1}^{K} \frac{\boldsymbol{\Phi}_{k}^{*} \boldsymbol{P}_{k} \boldsymbol{\Phi}_{k}^{T}}{\sigma^{2}}\right|
\end{aligned}
$$

where $\boldsymbol{P}_{k}$ is an $M \times M$ diagonal matrix whose diagonal entries are equal to those of $\boldsymbol{Q}_{M}^{H} E\left[\boldsymbol{x}_{k} \boldsymbol{x}_{k}^{T}\right] \boldsymbol{Q}_{M}$. The last inequality in (19) is due to the generalized Hadamard inequality [12], [15], which indicates that the determinant of a positive-definite matrix is upper-bounded by the product of the determinants of its diagonal blocks. 
Denote the $m$ th diagonal entry of $\boldsymbol{P}_{k}$ by $p_{k m}$. Since (19) holds with equality when $\boldsymbol{Q}_{M}^{H} E\left[\boldsymbol{x}_{k} \boldsymbol{x}_{k}^{T}\right] \boldsymbol{Q}_{M}$ is diagonal, substituting (19) into (14), we obtain

$$
\begin{aligned}
C_{\text {sum }} \leq & \lim _{M \rightarrow \infty} \max _{\sum_{m=1}^{M} p_{k m} \leq M P_{k}} \\
& \frac{1}{2 M} \sum_{m=1}^{M} \log _{2}\left[\left|\boldsymbol{I}_{N}+\sum_{k=1}^{K} \frac{\boldsymbol{\phi}_{k m}^{*} p_{k m} \boldsymbol{\phi}_{k m}^{T}}{\sigma^{2}}\right|\right] .
\end{aligned}
$$

When the signature sequences of the users are given, the sum capacity can be found by solving (20) with an iterative waterfilling algorithm [12], whose convergence is studied in [16]. It is easily seen that, given an arbitrary signature set and user delay profile, the sum capacity is usually not achieved by input signals with white spectra (i.e., $\boldsymbol{P}_{k}=P_{k} \boldsymbol{I}_{M}$ ).

In the following theorem, we give an upper bound on the sum capacity. The upper bound has the feature that it is not a function of the signature sequences of the users.

Theorem 2: Similar to [3], define user $k$ as oversized if

$$
P_{k}>\frac{\sum_{j=1}^{K} P_{j} 1_{P_{k}>P_{j}}}{N-\sum_{j=1}^{K} 1_{P_{k} \leq P_{j}}} .
$$

Denote the set of the oversized users as $\mathcal{K}$. The sum capacity is upper-bounded by

$$
\begin{aligned}
C_{\text {sum }} \leq \frac{N-|\mathcal{K}|}{2} \log _{2}(1+ & \left.\frac{\sum_{k \notin \mathcal{K}} P_{k}}{(N-|\mathcal{K}|) \sigma^{2}}\right) \\
& +\frac{1}{2} \sum_{k \in \mathcal{K}} \log _{2}\left(1+\frac{P_{k}}{\sigma^{2}}\right) .
\end{aligned}
$$

This upper bound equals the optimal sum capacity of the symbol synchronous system given in [3].

The proof of Theorem 2 is given in Appendix B.

Note that when the number of users $K$ is less than or equal to the spreading gain $N$, all the users are oversized. In the situation when $K>N$, it is possible that some or none of the users are oversized, depending on the signal powers.

\section{Optimal Signature SeQuences}

Although the upper bound given in (22) may not be achieved with an arbitrary signature sequence set, we show in this section that, it is indeed achievable when the signature sequences are optimally designed, given any arbitrary user delay profile and average power constraints.

\section{A. The Necessary and Sufficient Condition}

The following lemma shows the connection between the Frobenius norm, denoted by $\|\cdot\|_{F}$, and the eigenvalues of a symmetric matrix $\boldsymbol{A}$.

Lemma 1: Suppose $\boldsymbol{A}$ is an $N \times N$ positive-definite matrix that satisfies $\boldsymbol{A}^{H}=\boldsymbol{A}$. Suppose $\tilde{N} \leq N$ and $\lambda_{1}, \ldots, \lambda_{\tilde{N}}$ are all the nonzero eigenvalues of $\boldsymbol{A}$. If $\boldsymbol{A}$ is constrained such that $\operatorname{tr}(\boldsymbol{A})=P$, then

$$
\|A\|_{F}^{2} \geq \frac{P^{2}}{\tilde{N}}
$$

with equality if and only if $\lambda_{1}=\lambda_{2}=\cdots=\lambda_{\tilde{N}}=\frac{P}{\tilde{N}}$.

Proof:

$$
\begin{aligned}
\|\boldsymbol{A}\|_{F}^{2} & =\operatorname{tr}\left(\boldsymbol{A}^{H} \boldsymbol{A}\right)=\sum_{i=1}^{\tilde{N}} \lambda_{i}^{2} \geq \frac{\left(\sum_{i=1}^{\tilde{N}} \lambda_{i}\right)^{2}}{\tilde{N}} \\
& =\frac{(\operatorname{tr}(\boldsymbol{A}))^{2}}{\tilde{N}}=\frac{P^{2}}{\tilde{N}} .
\end{aligned}
$$

With the help of Lemma 1, we are now ready to present the necessary condition on the optimal signature sequence set, which guarantees that the sum capacity achieves the upper bound given in (22). Somewhat surprisingly, the condition becomes sufficient if the input signals are Gaussian with white spectra.

Theorem 3: If $\mathcal{K}$ is the set of oversized users, suppose that the user delay profile and the power constraints are given, assume that the signature sequences are normalized to $\boldsymbol{s}_{k}^{T} \boldsymbol{s}_{k}=1$, $\forall k$. The necessary conditions to achieve the sum capacity upper bound in (22) are that the input signals should be Gaussian and the following three conditions should hold.

- Condition 1: For all $k \in \mathcal{K}$ and $j \neq k$

$$
\begin{gathered}
\boldsymbol{s}_{k}^{L^{T}} \boldsymbol{s}_{j}^{L}+\boldsymbol{s}_{k}^{R^{T}} \boldsymbol{s}_{j}^{R}=0 \\
\boldsymbol{s}_{k}^{L^{T}} \boldsymbol{s}_{j}^{R}=0 \text { and } \boldsymbol{s}_{k}^{R^{T}} \boldsymbol{s}_{j}^{L}=0 .
\end{gathered}
$$

The input signal of user $k \in \mathcal{K}$ has white power spectrum.

- Condition 2:

$$
\sum_{k \notin \mathcal{K}} P_{k} \boldsymbol{s}_{k}^{L} \boldsymbol{s}_{k}^{R^{T}}=\mathbf{0}
$$

- Condition 3:

$$
\left\|\sum_{k \notin \mathcal{K}} P_{k}\left(\boldsymbol{s}_{k}^{L} \boldsymbol{s}_{k}^{L^{T}}+\boldsymbol{s}_{k}^{R} \boldsymbol{s}_{k}^{R^{T}}\right)\right\|_{F}^{2}=\frac{\left(\sum_{k \notin \mathcal{K}} P_{k}\right)^{2}}{N-|\mathcal{K}|} .
$$

Furthermore, under the assumption that user signals are white Gaussian, i.e., $E\left[\boldsymbol{x}_{k} \boldsymbol{x}_{k}^{T}\right]=P_{k} \boldsymbol{I}_{M}, \forall k$, the above three conditions become sufficient in the sense that if (25)-(27) hold, then the sum capacity of the system achieves the upper bound given in (22).

The proof of Theorem 3 is presented in Appendix C.

To be consistent with [3], we term the signature sequence set of the nonoversized users that satisfy conditions (25)-(27) the generalized asynchronous WBE (GAWBE) sequences.

Note that in the equal user power case, with condition (26), $\left\|P_{k} \sum_{k \notin K}\left(\boldsymbol{s}_{k}^{L} \boldsymbol{s}_{k}^{L^{T}}+\boldsymbol{s}_{k}^{R} \boldsymbol{s}_{k}^{R^{T}}\right)\right\|_{F}^{2}$ is proportional to the TSAC of the system, defined in [6], which is also the TSC when the user signals are symbol synchronous. Under the constraint of 
(26), Lemma 1 implies that minimizing the TSC or the TSAC results in the optimal signature sequences. ${ }^{4}$

It is worth pointing out that, although the capacity upper bound can be reached with white Gaussian inputs, white spectra of the input signals of the nonoversized users are not required for the sum capacity to reach its upper bound, even for the symbol synchronous case. Consider the conventional symbol synchronous two-user multiple-access channel with the observation of the symbol matched filter output given by $y=x_{1}+x_{2}+n$, where $E\left[x_{1}^{2}\right] \leq P_{1}, E\left[x_{2}^{2}\right] \leq P_{2}$ are the average power constraints, and $n$ is a zero mean Gaussian noise with variance $E\left[n^{2}\right]=\sigma^{2}$. Suppose that the power spectra of the two user signals are $S_{1}(\omega), S_{2}(\omega)$, respectively. Although it is shown that $S_{1}(\omega)=P_{1}$ and $S_{2}(\omega)=P_{2}$ maximizes the capacity region [12], the sum capacity of $C=\frac{1}{2} \log _{2}\left(1+\frac{P_{1}+P_{2}}{\sigma^{2}}\right)$ can be achieved by any spectra pair as long as $S_{1}(\omega)+S_{2}(\omega)=P_{1}+P_{2}$ is satisfied for all $\omega$.

\section{B. Construction of the Optimal Signature Sequences}

Assume that the input signals have white spectra. Although Theorem 3 gives the necessary and sufficient condition for the sum capacity to reach its upper bound, it can be seen that the conditions for the symbol asynchronous system are much stricter than the symbol synchronous case in general. Hence, given an arbitrary power constraint set and a user delay profile, it is natural to ask whether such optimal signature sequence set always exists. In this section, we provide an explicit algorithm to construct the optimal signature sequence set that satisfies all three conditions in Theorem 3. The construction algorithm guarantees that the upper bound in (22) is always achieved with independent and identically distributed (i.i.d.) Gaussian inputs.

First, we consider the situation when there is no oversized user, i.e., $\forall k$, the powers of all the users satisfy

$$
P_{k} \leq \frac{\sum_{j=1}^{K} P_{j} 1_{P_{k}>P_{j}}}{N-\sum_{j=1}^{K} 1_{P_{k} \leq P_{j}}} \leq \frac{\sum_{j=1}^{K} P_{j} 1_{P_{k}>P_{j}}}{N} \leq \frac{\sum_{j=1}^{K} P_{j}}{N} .
$$

Note that inequality (28) implies $K>N$. The situation when we have oversized users, which includes the situation of $K \leq$ $N$, is considered later in this section.

Our purpose is to construct a signature sequence set such that $\boldsymbol{s}_{k}^{R}=\mathbf{0}$ for all $k$. Although this guarantees that the requirement in (26) is met, unfortunately, meeting the requirement in (27) with such a strict constraint is not always possible for a general user delay profile. We use the time labeling idea presented in [17], that enables us to work with other $N-1$ equivalent delay profiles, even when the user delay profile is given. This significantly increases our freedom in the signature design. We will show that, among all the $N$ equivalent user delay profiles, there always exists one that allows us to construct an optimal signature sequence set that simultaneously satisfies $\boldsymbol{s}_{k}^{R}=\mathbf{0}, \forall k$ and the condition in (27).

Arrange the users in increasing order of their delays, i.e., $c_{1} \leq$ $c_{2} \leq \cdots \leq c_{K}$. Since all $c_{k}$ take values in $[0, N-1]$, we can divide the users into $N$ groups, denoted by $G_{1}, G_{2}, \ldots, G_{N}$,

\footnotetext{
${ }^{4} \mathrm{As}$ shown in [6, Theorem 3], when the TSAC lower bound is achieved, both (26) and (27) are satisfied, and hence the signature sequences are optimal according to Theorem 3 in this paper.
}

such that the users in the same group are symbol synchronous. Specifically, the delay of the users satisfy

$$
c_{k}=i-1, \quad \text { if } k \in G_{i} .
$$

The aggregate signal power in group $G_{i}$ is denoted by

$$
P_{G_{i}}=\sum_{k \in G_{i}} P_{k}
$$

It is possible that some of the groups are empty, depending on the user delay profile.

Note that (29) is based on the assumption that the users in group $G_{1}$ have zero delays. Different choices of $G_{1}$ determine the symbol delay value $c_{k}$ for all $k$. Here we refer to the choice of $G_{1}$ as the definition of the time labeling of the system. In the literature of symbol asynchronous CDMA, usually, such a definition is either chosen according to the physical arrival of the user signals [1], or it is chosen arbitrarily [6]. For example, in [6], an arbitrary $N$ chip duration is chosen. For any user $k$, there is one and only one symbol starting time located within this $N$ chip duration, taking values in $[0, N-1]$. The time label of user $k$ is defined such that the symbol before the symbol starting time contributes to the $n$th symbol and the symbol after the symbol starting time contributes to the $(n+1)$ th symbol [6, Fig. 2]. Although time labeling does not affect the capacity region of the system for any given signature sequence set, different choices of the $N$ chip duration result in different signature design problems [6]. In fact, the choice of the time labeling is also the key factor in many other research problems in asynchronous CDMA, such as the design of the decision feedback multiuser detector, as shown in [17].

Suppose that we pick an arbitrary time labeling and define it as $T_{1}=\left[G_{1}, G_{2}, \ldots, G_{N}\right]$. Now, without changing the physical signals, if we change the time labels and define the users in group $G_{N}$ as the zero-delayed users, we can reorder the groups chronologically according to the new time labels and get another equivalent group ordering, $T_{2}=$ $\left[G_{N}, G_{1}, G_{2}, \ldots, G_{N-1}\right]$. Overall, there are $N$ different time labelings, and each time labeling is uniquely determined by the corresponding chronological order of the groups. We say that time labeling $T_{2}$ is obtained from $T_{1}$ through a backward rotation.

The following theorem shows that, among the $N$ different time labelings, there is one time labeling that possesses a special property, which is the key feature that ensures the existence of the optimal signature sequence set.

Theorem 4: Assume that there are no oversized users. Among all the time labelings, there exists one time labeling $\hat{T}=\left[\hat{G}_{1}, \hat{G}_{2}, \ldots, \hat{G}_{N}\right]$, such that $\forall 1 \leq g \leq N$, the signal powers satisfy

$$
\sum_{j=1}^{g} P_{\hat{G}_{j}} \geq \frac{g \sum_{k=1}^{K} P_{k}}{N} .
$$

The proof of Theorem 4 is presented in Appendix D.

Now, consider the time labeling $\hat{T}$, and arrange the users and the groups in the chronological order of their symbol delays. For notational simplicity, we still term these 
groups $G_{1}, G_{2}, \ldots, G_{N}$. Consider the signature matrix $\tilde{\boldsymbol{S}}=$ $\left[\begin{array}{lll}\tilde{\boldsymbol{s}}_{1} & \ldots & \tilde{\boldsymbol{s}}_{K}\end{array}\right]$ where

$$
\tilde{\boldsymbol{s}}_{k}=\left[\tilde{\boldsymbol{s}}_{k}^{R^{T}}, \tilde{\boldsymbol{s}}_{k}^{L^{T}}\right]^{T}
$$

is constructed by stacking the right signature sequence and the left signature sequence of user $k$ together. Here, the left signature sequence $\tilde{\boldsymbol{s}}_{k}^{L}$ and the right signature sequence $\tilde{\boldsymbol{s}}_{k}^{R}$ are defined as in (1) according to the symbol delay $c_{k}$ of user $k$ in time labeling $\hat{T}$. Note that in the special case when user powers are equal, Theorem 4 indicates that, in time labeling $\hat{T}$, all the right signature sequences of the users are located within the upper-right triangular part of the matrix $\tilde{\boldsymbol{S}}$. Next, we show that one can always construct a GAWBE sequence set, such that all the components of the right signature sequences are 0 's.

\section{Construction of the GAWBE sequence set:}

- Step 1: Initialize $\boldsymbol{h}_{0}$ as a $K \times 1$ column vector whose first $N$ components have identical values of $\frac{\sum_{k=1}^{K} P_{k}}{N}$ and other components are 0 , i.e.,

$$
h_{0}=[\overbrace{\frac{\sum_{k=1}^{K} P_{k}}{N}, \ldots, \frac{\sum_{k=1}^{K} P_{k}}{N}}^{N \text { items }}, 0, \ldots, 0]^{T} .
$$

Initialize matrix $\boldsymbol{H}_{0}=\operatorname{diag}\left(\boldsymbol{h}_{0}\right)$. Let $i=1$.

- Step 2: Construct a $K \times 1$ column vector $\boldsymbol{h}_{i}$ such that the following properties are satisfied.

- Property 1: All the components of $h_{i}$ are nonnegative.

- Property 2: The first $N-i$ components of $\boldsymbol{h}_{i}$ have value $\frac{\sum_{k=1}^{K} P_{k}}{N}$.

- Property 3: The last $\sum_{j=N-i+1}^{N}\left|G_{j}\right|$ components of $\boldsymbol{h}_{i}$ are the powers of users in groups $G_{N-i+1}, \ldots, G_{N}$, respectively, in the same order.

- Property 4: The components located between the $(N-i)$ th and the $\left(\sum_{j=1}^{N-i}\left|G_{j}\right|+1\right)$ th components satisfy

$$
\left[\boldsymbol{h}_{i}\right]_{j} \leq\left[\boldsymbol{h}_{i-1}\right]_{j}, \quad \forall N-i<j \leq \sum_{j=1}^{N-i}\left|G_{j}\right| .
$$

If we denote the powers of the users in group $G_{i}$ as $P_{1}^{\left(G_{i}\right)}, \ldots, P_{\left|G_{i}\right|}^{\left(G_{i}\right)}, \boldsymbol{h}_{i}$ can be described as in (34) at the bottom of the page, where the components marked with "?" can be arbitrary so long as they satisfy the inequality in (33).

Note that such an $h_{i}$ vector always exists since from (31) we have,

$$
\begin{aligned}
& \sum_{j=1}^{N-i} P_{G_{j}} \geq \frac{(N-i) \sum_{k=1}^{K} P_{k}}{N} \\
& \sum_{j=1}^{N} P_{G_{j}}=\sum_{k=1}^{K} P_{k}
\end{aligned}
$$

which yields

$$
\sum_{j=N-i+1}^{N} P_{G_{j}} \leq \frac{i \sum_{k=1}^{K} P_{k}}{N} .
$$

Since $\boldsymbol{h}_{i-1}$ and $\boldsymbol{h}_{i}$ satisfy the inequality (33), by following the method in Section IV of [3], we can construct a unitary matrix $U_{N-i+1}$, such that the diagonal components of $\boldsymbol{U}_{N-i+1}^{T} \boldsymbol{H}_{i-1} \boldsymbol{U}_{N-i+1}$ are exactly the components of $\boldsymbol{h}_{i}$, in the same order. We define $\boldsymbol{H}_{\boldsymbol{i}}=\boldsymbol{U}_{N-i+1}^{T} \boldsymbol{H}_{i-1} \boldsymbol{U}_{N-i+1}$.

- Step 3: If $i<N$, let $i=i+1$ and goto Step 2. Otherwise, go to Step 4.

- Step 4: Define $\boldsymbol{U}=\boldsymbol{U}_{N} \boldsymbol{U}_{N-1} \ldots \boldsymbol{U}_{1}$, and define the matrix that contains the first $N$ rows of $\boldsymbol{U}$ as $\boldsymbol{V}$. We can then construct the signature sequence matrix as

$$
\tilde{\boldsymbol{S}}=\sqrt{\frac{\sum_{k=1}^{K} P_{k}}{N}} \boldsymbol{V} \operatorname{diag}\left(\frac{1}{\sqrt{P_{1}}}, \ldots, \frac{1}{\sqrt{P_{K}}}\right) .
$$

Theorem 5: The GAWBE signature sequences constructed via the above procedure satisfy the conditions (26) and (27) in Theorem 3, for any time labeling.

The proof of Theorem 5 is presented in Appendix E.

Compared with the optimal signature sequence construction in the symbol synchronous case [3], the key steps that result in $\boldsymbol{s}_{k}^{R}=\mathbf{0}$, for all $k$ in time labeling $\hat{T}$, are that, first, we operate on the diagonal components of the $\boldsymbol{H}_{i}$ matrices in a specific order, and second, the time labeling $\hat{T}$ satisfies the property shown in (31).

$$
\boldsymbol{h}_{i}=[\overbrace{\overbrace{\sum_{k=1}^{K} P_{k}}^{N}, \ldots, \frac{\sum_{k=1}^{K} P_{k}}{N}, ?, \ldots, ?, P_{1}^{\left(G_{N-i+1}\right)}, P_{2}^{\left(G_{N-i+1}\right)}, \ldots, P_{\left|G_{N}\right|}^{\left(G_{N}\right)}}^{N-i \text { items }}]^{T}
$$


In the general case, where there are $|\mathcal{K}|$ oversized users, we arrange the users such that users $1, \ldots, K-|\mathcal{K}|$ are nonoversized and users $K-|\mathcal{K}|+1, \ldots, K$ are oversized. Now, we partition the signature sequence matrix $\tilde{\boldsymbol{S}}$ as

$$
\tilde{\boldsymbol{S}}=\left[\begin{array}{cc}
\tilde{\boldsymbol{S}}_{K-|\mathcal{K}|} & \mathbf{0} \\
\mathbf{0} & \boldsymbol{I}_{|\mathcal{K}|}
\end{array}\right]
$$

The signature sequence of user $k>K-|\mathcal{K}|$ is designed as $\tilde{\boldsymbol{s}}_{k}=\boldsymbol{e}_{k}$, where $\boldsymbol{e}_{k}$ is the column vector whose $k$ th component is 1 and all other components are 0 . The signature sequences of the nonoversized users are designed such that $\tilde{s}_{k j}=0$, $\forall k \leq K-|\mathcal{K}|$, and $j>N-|\mathcal{K}|$. Consequently, (25) is satisfied irrespective of the values of the components in the upper-left block matrix $\tilde{\boldsymbol{S}}_{K-|\mathcal{K}|}$.

Now consider the subblock matrix $\tilde{\boldsymbol{S}}_{K-|\mathcal{K}|}$ with dimension $(N-|\mathcal{K}|) \times(K-|\mathcal{K}|)$ in (38). The signature sequence design of $\tilde{\boldsymbol{S}}_{K-|\mathcal{K}|}$ is equivalent to that of a $K-|\mathcal{K}|$ user system with spreading factor of $N-|\mathcal{K}|$, where no oversized users are present. Therefore, we can assign the GAWBE sequence set for $\tilde{\boldsymbol{S}}_{K-|\mathcal{K}|}$, which completes the optimal sequence construction of the $K$-user system.

Next we will give a simple example to clarify the construction scheme. Suppose we have four users, where the users have powers of $1,1,1,2$ units and delays $0,0,1,2$ chips, respectively. In this case, user 4 is the only oversized user. Therefore, we first design the signature sequence matrix as in (38)

$$
\tilde{\boldsymbol{S}}=\left[\begin{array}{cc}
\tilde{\boldsymbol{S}}_{3} & \mathbf{0} \\
\mathbf{0} & 1
\end{array}\right] \text {. }
$$

Now, consider the subblock $\tilde{\boldsymbol{S}}_{3}$. Divide the users into two groups according to their delays, i.e., $G_{1}=[1,2]$ and $G_{2}=[3]$. Since $P_{1}+P_{2}=2>\frac{1}{2}\left(P_{1}+P_{2}+P_{3}\right)$, inequality (31) is satisfied. Hence, we just work with the current time labeling.

In the construction of the GAWBE sequence set, we initialize $\boldsymbol{h}_{0}=\left[\frac{3}{2}, \frac{3}{2}, 0\right]$, and $\boldsymbol{H}_{0}=\operatorname{diag}\left(\boldsymbol{h}_{0}\right)$. Choose $\boldsymbol{h}_{1}=\left[\frac{3}{2}, \frac{1}{2}, 1\right]$, which satisfies $\left[\boldsymbol{h}_{1}\right]_{3}=P_{3},\left[\boldsymbol{h}_{1}\right]_{1}=\frac{P_{1}+P_{2}+P_{3}}{2}$, and $\left[\boldsymbol{h}_{1}\right]_{2} \leq\left[\boldsymbol{h}_{0}\right]_{2}$. By following the method in [3], we construct

Consequently

$$
\boldsymbol{U}_{2}=\left[\begin{array}{ccc}
1 & 0 & 0 \\
0 & \sqrt{\frac{1}{3}} & \sqrt{\frac{2}{3}} \\
0 & -\sqrt{\frac{2}{3}} & \sqrt{\frac{1}{3}}
\end{array}\right] .
$$

$$
\boldsymbol{H}_{1}=\boldsymbol{U}_{2}^{T} \boldsymbol{H}_{0} \boldsymbol{U}_{2}=\left[\begin{array}{ccc}
\frac{3}{2} & 0 & 0 \\
0 & \frac{1}{2} & \sqrt{\frac{1}{2}} \\
0 & \sqrt{\frac{1}{2}} & 1
\end{array}\right]
$$

where the diagonal components of $\boldsymbol{H}_{1}$ equal the components of $\boldsymbol{h}_{1}$ in the same order.

Next, we choose $\boldsymbol{h}_{2}=[1,1,1]$, and construct

$$
U_{1}=\left[\begin{array}{ccc}
\sqrt{\frac{1}{2}} & \sqrt{\frac{1}{2}} & 0 \\
-\sqrt{\frac{1}{2}} & \sqrt{\frac{1}{2}} & 0 \\
0 & 0 & 1
\end{array}\right]
$$

and have

$$
\boldsymbol{H}_{2}=\boldsymbol{U}_{1}^{T} \boldsymbol{H}_{1} \boldsymbol{U}_{1}=\left[\begin{array}{ccc}
1 & \frac{1}{2} & -\frac{1}{2} \\
\frac{1}{2} & 1 & \frac{1}{2} \\
-\frac{1}{2} & \frac{1}{2} & 1
\end{array}\right] .
$$

Now define $\boldsymbol{U}=\boldsymbol{U}_{2} \boldsymbol{U}_{1}$, and define $\boldsymbol{V}$ as the matrix that contains the first two rows of $\boldsymbol{U}$.

$$
V=\left[\begin{array}{ccc}
\sqrt{\frac{1}{2}} & \sqrt{\frac{1}{2}} & 0 \\
-\sqrt{\frac{1}{6}} & \sqrt{\frac{1}{6}} & \sqrt{\frac{2}{3}}
\end{array}\right] .
$$

Therefore, the subblock $\tilde{\boldsymbol{S}}_{3}$ is constructed as

$$
\begin{aligned}
\tilde{\boldsymbol{S}}_{3} & =\sqrt{\frac{P_{1}+P_{2}+P_{3}}{2}} \boldsymbol{V} \operatorname{diag}\left(\frac{1}{\sqrt{P_{1}}}, \frac{1}{\sqrt{P_{2}}}, \frac{1}{\sqrt{P_{3}}}\right) \\
& =\left[\begin{array}{ccc}
\frac{\sqrt{3}}{2} & \frac{\sqrt{3}}{2} & 0 \\
-\frac{1}{2} & \frac{1}{2} & 1
\end{array}\right] .
\end{aligned}
$$

Combined with (39), we get the signature sequence matrix $\tilde{\boldsymbol{S}}$ as

$$
\tilde{\boldsymbol{S}}=\left[\begin{array}{cccc}
\frac{\sqrt{3}}{2} & \frac{\sqrt{3}}{2} & 0 & 0 \\
-\frac{1}{2} & \frac{1}{2} & 1 & 0 \\
0 & 0 & 0 & 1
\end{array}\right] .
$$

It is easy to verify that $\tilde{\boldsymbol{S}}$ satisfies the requirements in Theorem 3.

\section{CONCLUSION}

The optimal real-valued signature sequences that maximize the sum capacity of a symbol asynchronous (but chip synchronous) CDMA system are characterized in this paper. By assigning orthogonal signature sequences to the oversized users, and assigning a GAWBE signature sequence set to the nonoversized users, the asynchronous system achieves the same sum capacity as the synchronous system with the same user power constraints. An explicit method is provided for the construction of the optimal signature sequence set. This resolves the existence of the optimal signature sequences for arbitrary user delay profiles and average power constraints. Although the results presented in this paper give insights to the signature design problem for practical systems, the optimal signature design problem that considers chip asynchronous systems and finite-valued signature sequences remains open.

\section{APPENDIX A \\ PROOF OF THEOREM 1}

Proof: Consider user $k \in J$. Since $E\left[\boldsymbol{x}_{k} \boldsymbol{x}_{k}^{T}\right]$ is positive definite, we can find an upper triangular matrix $\boldsymbol{F}_{k}$, such that

$$
E\left[\boldsymbol{x}_{k} \boldsymbol{x}_{k}^{T}\right]=\boldsymbol{F}_{k} \boldsymbol{F}_{k}^{T}
$$

is the Cholesky decomposition of $E\left[\boldsymbol{x}_{k} \boldsymbol{x}_{k}^{T}\right]$. Since $\hat{\boldsymbol{S}}_{k}^{T} \hat{\boldsymbol{S}}_{k}$ is block circulant and $\boldsymbol{S}_{k}^{T} \boldsymbol{S}_{k}$ is asymptotically block circulant, it is easy to see that eigenvalues of both $\hat{\boldsymbol{S}}_{k}^{T} \hat{\boldsymbol{S}}_{k}$ and $\boldsymbol{S}_{k}^{T} \boldsymbol{S}_{k}$ are bounded. Due to the fact that the average power per symbol of the signal of user $k$ is bounded, as $M \rightarrow \infty$, the operator norm of $E\left[x_{k} x_{k}^{T}\right]$ is uniformly bounded. Consequently, the operator norms of both $\hat{\boldsymbol{S}}_{k} E\left[\boldsymbol{x}_{k} \boldsymbol{x}_{k}^{T}\right] \hat{\boldsymbol{S}}_{k}^{T}$ and $\boldsymbol{S}_{k} E\left[\boldsymbol{x}_{k} \boldsymbol{x}_{k}^{T}\right] \boldsymbol{S}_{k}^{T}$ are uniformly bounded. Since $\boldsymbol{S}_{k} \boldsymbol{F}_{k}$ and $\hat{\boldsymbol{S}}_{k} \boldsymbol{F}_{k}$ differ only on the $N \times 1$ column vector located on the upper-right corner, it is easy to see that

$$
\begin{aligned}
\lim _{M \rightarrow \infty}\left\{\frac{1}{M} \operatorname{tr}\right. & {\left[\left(\boldsymbol{S}_{k} \boldsymbol{F}_{k} \boldsymbol{F}_{k}^{T} \boldsymbol{S}_{k}^{T}-\hat{\boldsymbol{S}}_{k} \boldsymbol{F}_{k} \boldsymbol{F}_{k}^{T} \hat{\boldsymbol{S}}_{k}^{T}\right)^{T}\right.} \\
& \left.\left.\left(\boldsymbol{S}_{k} \boldsymbol{F}_{k} \boldsymbol{F}_{k}^{T} \boldsymbol{S}_{k}^{T}-\hat{\boldsymbol{S}}_{k} \boldsymbol{F}_{k} \boldsymbol{F}_{k}^{T} \hat{\boldsymbol{S}}_{k}^{T}\right)\right]\right\}^{\frac{1}{2}}=0 .
\end{aligned}
$$


This shows that $\hat{\boldsymbol{S}}_{k} E\left[\boldsymbol{x}_{k} \boldsymbol{x}_{k}^{T}\right] \hat{\boldsymbol{S}}_{k}^{T}$ and $\boldsymbol{S}_{k} E\left[\boldsymbol{x}_{k} \boldsymbol{x}_{k}^{T}\right] \boldsymbol{S}_{k}^{T}$ are asymptotically equivalent. Theorem 1 then follows from the property of asymptotically equivalent matrices presented in [13, Theorem 2.1].

\section{APPENDIX B \\ PROOF OF THEOREM 2}

Proof: Without loss of generality, let us assume that the user powers satisfy $P_{1} \geq P_{2} \geq \cdots \geq P_{K}$. According to Theorem 1, to obtain an upper bound on the sum capacity, we can choose $\hat{\boldsymbol{S}}_{k}$ and $E\left[\boldsymbol{x}_{k} \boldsymbol{x}_{k}^{T}\right], \forall k$, under the power constraints (5), such that the following quantity is maximized:

$$
\frac{1}{2 M} \log _{2}\left[\left|\boldsymbol{I}_{M N}+\sum_{k=1}^{K} \frac{\hat{\boldsymbol{S}}_{k} E\left[\boldsymbol{x}_{k} \boldsymbol{x}_{k}^{T}\right] \hat{\boldsymbol{S}}_{k}^{T}}{\sigma^{2}}\right|\right] .
$$

Define $p_{1 m}=E\left[x_{k}(m)^{2}\right]$; define the vector $\boldsymbol{p}_{1}=$ $\left[p_{11}, \ldots, p_{1 M}\right]^{T}$; and define the $M K$-length column vector $\boldsymbol{p}$ such that $\boldsymbol{p}=\left[\boldsymbol{p}_{1}^{T}, \ldots, \boldsymbol{p}_{K}^{T}\right]^{T}$. Now, let $\mathcal{N}_{P}$ be the set of all $\boldsymbol{p}$ vectors satisfying the power constraint (5). Let $\boldsymbol{\lambda}=$ $\left[\lambda_{1}, \ldots, \lambda_{M N}\right]^{T}$ be the eigenvalues of $\sum_{k=1}^{K} \hat{\boldsymbol{S}}_{k} E\left[\boldsymbol{x}_{k} \boldsymbol{x}_{k}^{T}\right] \hat{\boldsymbol{S}}_{k}^{T}$. Define $\mathcal{N}_{\lambda}$ as the set of eigenvalue vectors, such that for any $\boldsymbol{\lambda} \in \mathcal{N}_{\lambda}$, there exists a $\boldsymbol{p} \in \mathcal{N}_{P}$, and $\left[\lambda_{1}, \ldots, \lambda_{M N}, 0, \ldots, 0\right]^{T}$ majorizes $\boldsymbol{p}$ (see the definition of majorization in [3], [18]). Since $\boldsymbol{S}_{k}$ is replaced by $\hat{\boldsymbol{S}}_{k}, \forall k$, we can view the $K$-user $M$-symbol asynchronous system as an $M K$-user symbol synchronous system. With a simple extension to [3, Lemma 3.1], we can see that for all $\boldsymbol{\lambda} \in \mathcal{N}_{\lambda}, \boldsymbol{\lambda}$ majorizes $\boldsymbol{\lambda}^{*}$, where $\boldsymbol{\lambda}^{*}$ is given by (49) at the bottom of the page. Since

$$
f(\boldsymbol{\lambda})=\sum_{i=1}^{M N} \log _{2}\left(1+\frac{\lambda_{i}}{\sigma^{2}}\right)
$$

is a strictly Schur-concave function, according to [18, Theorem 3.C.1], we have

$$
\begin{aligned}
f(\boldsymbol{\lambda})= & \frac{1}{2 M} \log _{2}\left[\left|\boldsymbol{I}_{M N}+\sum_{k=1}^{K} \frac{\hat{\boldsymbol{S}}_{k} E\left[\boldsymbol{x}_{k} \boldsymbol{x}_{k}^{T}\right] \hat{\boldsymbol{S}}_{k}^{T}}{\sigma^{2}}\right|\right] \\
\leq & f\left(\boldsymbol{\lambda}^{*}\right) \\
= & \frac{N-|\mathcal{K}|}{2} \log _{2}\left(1+\frac{\sum_{k \notin \mathcal{K}} P_{k}}{(N-|\mathcal{K}|) \sigma^{2}}\right) \\
& +\frac{1}{2} \sum_{k \in \mathcal{K}} \log _{2}\left(1+\frac{P_{k}}{\sigma^{2}}\right)
\end{aligned}
$$

which gives the desired result.

\section{APPENDIX C PROOF OF THEOREM 3}

Proof: We first consider the sufficiency part of the theorem.

Assume that $E\left[\boldsymbol{x}_{k} \boldsymbol{x}_{k}^{T}\right]=P_{k} \boldsymbol{I}_{M}, \forall k$, we prove that the sum capacity achieves the upper bound if (25)-(27) are satisfied.

Consider the sum capacity given in (14), if $E\left[x_{k} x_{k}^{T}\right]=$ $P_{k} \boldsymbol{I}_{M}, \forall k$, we have

$$
\sum_{k=1}^{K} \hat{\boldsymbol{S}}_{k} E\left[\boldsymbol{x}_{k} \boldsymbol{x}_{k}^{T}\right] \hat{\boldsymbol{S}}_{k}^{T}=\sum_{k=1}^{K} P_{k} \hat{\boldsymbol{S}}_{k} \hat{\boldsymbol{S}}_{k}^{T} .
$$

Note that for any user $k \in \mathcal{K}$, condition (25) ensures that the columns of $\hat{\boldsymbol{S}}_{k}$ are orthogonal to the columns of $\hat{\boldsymbol{S}}_{j}, \forall j \neq k$. Therefore, the matrix $\sum_{k=1}^{K} P_{k} \hat{\boldsymbol{S}}_{k} \hat{\boldsymbol{S}}_{k}^{T}$ has $M$ eigenvalues with the values of $P_{k}$, for any $k \in \mathcal{K}$.

Now consider the $M N \times M N$ matrix $\sum_{k \notin \mathcal{K}} P_{k} \hat{\boldsymbol{S}}_{k} \hat{\boldsymbol{S}}_{k}^{T}$. With condition (26), it is easy to verify that $\sum_{k \notin \mathcal{K}} P_{k} \hat{\boldsymbol{S}}_{k} \hat{\boldsymbol{S}}_{k}^{T}$ is block diagonal, and each of the $M$ diagonal blocks equals $\sum_{k \notin K} P_{k}\left(\boldsymbol{s}_{k}^{L} \boldsymbol{s}_{k}^{L T}+\boldsymbol{s}_{k}^{R} \boldsymbol{s}_{k}^{R^{T}}\right)$. Since with condition (25), $\sum_{k \notin \mathcal{K}} P_{k} \hat{\boldsymbol{S}}_{k} \hat{\boldsymbol{S}}_{k}^{T}$ has at most $M(N-|\mathcal{K}|)$ nonzero eigenvalues, according to Lemma 1, condition (27) ensures that all the nonzero eigenvalues of $\sum_{k \notin \mathcal{K}} P_{k} \hat{\boldsymbol{S}}_{k} \hat{\boldsymbol{S}}_{k}^{T}$ are equal to $\frac{\sum_{k \notin \mathcal{K}} P_{k}}{N-|\mathcal{K}|}$ In other words, the columns of $\hat{\boldsymbol{S}}_{k}, \forall k \notin \mathcal{K}$, form a GWBE sequence set. The result that the sum capacity achieves the upper bound given in (22) then follows.

Next, we consider the necessity part of the theorem.

Without loss of generality, suppose that we have $|\mathcal{K}|$ oversized users, with $P_{1} \geq P_{2} \geq \cdots \geq P_{|\mathcal{K}|}$, and for the nonoversized users, we assume that their symbol delays satisfy $c_{|\mathcal{K}|+1} \leq c_{|\mathcal{K}|+2} \leq \cdots \leq c_{K}$. If the sum capacity of the system achieves the upper bound in (22), from the proof of Theorem 2, asymptotically, the $M$ largest eigenvalues of $\sum_{k=1}^{K} \hat{\boldsymbol{S}}_{k} E\left[\boldsymbol{x}_{k} \boldsymbol{x}_{k}^{T}\right] \hat{\boldsymbol{S}}_{k}^{T}$ are equal almost surely to $P_{1}$. Consequently, all of the $M$ nonzero eigenvalues of the matrix $\hat{\boldsymbol{S}}_{1} E\left[\boldsymbol{x}_{1} \boldsymbol{x}_{1}^{T}\right] \hat{\boldsymbol{S}}_{1}^{T}$ are equal to $P_{1}$ almost surely. This gives that $E\left[\boldsymbol{x}_{1} \boldsymbol{x}_{1}^{T}\right]=P_{1} \boldsymbol{I}_{M}$, asymptotically, and the columns of $\hat{\boldsymbol{S}}_{1}$ are orthogonal to the columns of $\hat{\boldsymbol{S}}_{j}$, as long as $j \neq 1$. Therefore, condition (25) holds for $k=1$. By carrying out this analysis iteratively, it is easy to see that (25) holds for all $k \in \mathcal{K}$.

Now, consider only the users $k \notin \mathcal{K}$. According to (19), the sum capacity is achieved only when $\boldsymbol{Q}_{M}^{H} E\left[\boldsymbol{x}_{k} \boldsymbol{x}_{k}^{T}\right] \boldsymbol{Q}_{M}$ is asymptotically diagonal. Denote the $m$ th diagonal component of $\boldsymbol{Q}_{M}^{H} E\left[\boldsymbol{x}_{k} \boldsymbol{x}_{k}^{T}\right] \boldsymbol{Q}_{M}$ by $p_{k m}$. From the proof of Theorem 2 and (20), as $M \rightarrow \infty$, the matrix $\sum_{k \notin \mathcal{K}} \boldsymbol{\phi}_{k m}^{*} p_{k m} \boldsymbol{\phi}_{k m}^{T}$ must have $N-|\mathcal{K}|$ eigenvalues of $\frac{\sum_{k \notin \mathcal{K}} P_{k}}{N-|\mathcal{K}|}$ and all other $|\mathcal{K}|$ eigenvalues must be equal to 0 . Due to the fact that the signature

$$
\lambda^{*}=[\overbrace{P_{1}, \ldots, P_{1}}^{M \text { items }}, \ldots, \overbrace{P_{|\mathcal{K}|}, \ldots, P_{|\mathcal{K}|}}^{M \text { items }}, \frac{\overbrace{\sum_{k \notin \mathcal{K}} P_{k}}^{M(N-|\mathcal{K}|)}, \ldots, \frac{\sum_{k \notin \mathcal{K}} P_{k}}{N-|\mathcal{K}|}}{N-|\mathcal{K}|}]^{T} .
$$


sequences are real valued and satisfy condition (25), we can find $N-|\mathcal{K}|$ real-valued eigenvectors $\boldsymbol{u}_{1}, \ldots, \boldsymbol{u}_{N-|\mathcal{K}|}$ such that $\forall 1 \leq i \leq N-|\mathcal{K}|$

$$
\boldsymbol{u}_{i}^{T}\left(\sum_{k \notin \mathcal{K}} \boldsymbol{\phi}_{k m}^{*} p_{k m} \boldsymbol{\phi}_{k m}^{T}\right) \boldsymbol{u}_{i}=\frac{\sum_{k \notin \mathcal{K}} P_{k}}{N-|\mathcal{K}|} .
$$

In other words, matrix $\sum_{k \notin \mathcal{K}} \boldsymbol{\phi}_{k m}^{*} p_{k m} \boldsymbol{\phi}_{k m}^{T}$ has real-valued eigenvectors and real-valued eigenvalues. Therefore, all the components of $\sum_{k \notin \mathcal{K}} \boldsymbol{\phi}_{k m}^{*} p_{k m} \boldsymbol{\phi}_{k m}^{T}$ are real valued.

However, from (18), we know that

$$
\begin{aligned}
\sum_{k \notin \mathcal{K}} \boldsymbol{\phi}_{k m}^{*} p_{k m} \boldsymbol{\phi}_{k m}^{T}= & \sum_{k \notin \mathcal{K}} p_{k m}\left(\boldsymbol{s}_{k}^{L} \boldsymbol{s}_{k}^{R^{T}} e^{-j \frac{2 \pi(m-1)}{M}}\right. \\
& \left.+\boldsymbol{s}_{k}^{R} \boldsymbol{s}_{k}^{L^{T}} e^{j \frac{2 \pi(m-1)}{M}}\right) \\
& +\sum_{k \notin \mathcal{K}} p_{k m}\left(\boldsymbol{s}_{k}^{L} \boldsymbol{s}_{k}^{L^{T}}+\boldsymbol{s}_{k}^{R} \boldsymbol{s}_{k}^{R^{T}}\right) .
\end{aligned}
$$

Since the symbol delays of the nonoversized users satisfy $c_{|\mathcal{K}|+1} \leq c_{|\mathcal{K}|+2} \leq \cdots \leq c_{K}$, it is easy to see that $\sum_{k \notin \mathcal{K}} p_{k m} \boldsymbol{s}_{k}^{L} \boldsymbol{s}_{k}^{R}{ }^{T}$ is a strict lower triangular matrix. Therefore, except for a finite number of $m$ 's, in order to have only real-valued components on the right-hand side of (54), we must have

$$
\sum_{k \notin \mathcal{K}} p_{k m} \boldsymbol{s}_{k}^{L} \boldsymbol{s}_{k}^{R^{T}}=\mathbf{0}
$$

From the fact that matrix $\sum_{k \notin \mathcal{K}} \boldsymbol{\phi}_{k m}^{*} p_{k m} \boldsymbol{\phi}_{k m}^{T}$ has $N-|\mathcal{K}|$ eigenvalues of $\frac{\sum_{k \notin \mathcal{K}} P_{k}}{N-|\mathcal{K}|}$ and all other $|\mathcal{K}|$ eigenvalues equal to 0 , according to Lemma 1 and (55), we have

$$
\left\|\sum_{k \notin \mathcal{K}} p_{k m}\left(\boldsymbol{s}_{k}^{L} \boldsymbol{s}_{k}^{L^{T}}+\boldsymbol{s}_{k}^{R} \boldsymbol{s}_{k}^{R^{T}}\right)\right\|_{F}^{2}=\frac{\left(\sum_{k \notin \mathcal{K}} P_{k}\right)^{2}}{N-|\mathcal{K}|} .
$$

Conditions (26) and (27) can then be derived from (55) and (56) using the fact that $\sum_{m=1}^{M} p_{k m}=M P_{k}$, for all $k$.

\section{APPENDIX D \\ PROOF OF THEOREM 4}

Proof: Note that since we assume that there are no oversized users, according to (28), when $g=N$, inequality (31) is always satisfied.

We start with time labeling $T_{1}=\left[G_{1}, G_{2}, \ldots, G_{N}\right]$. Suppose that $T_{1}$ does not satisfy (31). We can find the minimum index $g_{\min }\left(T_{1}\right)$, such that (31) is satisfied for all $g<g_{\min }\left(T_{1}\right)$, but

$$
\sum_{j=1}^{g_{\min }} P_{G_{j}}<\frac{g_{\min } \sum_{k=1}^{K} P_{k}}{N} .
$$

Also, we can find the maximum index $g_{\max }\left(T_{1}\right)<N$, such that (31) is satisfied for all $g>g_{\max }\left(T_{1}\right)$, but

$$
\sum_{j=1}^{g_{\max }} P_{G_{j}}<\frac{g_{\max } \sum_{k=1}^{K} P_{k}}{N} .
$$

Since by assumption

$$
\sum_{j=1}^{g_{\max }+m} P_{G_{j}} \geq \frac{\left(g_{\max }+m\right) \sum_{k=1}^{K} P_{k}}{N}, \quad \forall m \leq N-g_{\max }
$$

we can see from (58) and (59) that the groups $G_{g_{\max }+1}, \ldots, G_{N}$ satisfy

$$
\sum_{j=g_{\max }+1}^{g_{\max }+m} P_{G_{j}} \geq \frac{m \sum_{k=1}^{K} P_{k}}{N}, \quad \forall m \leq N-g_{\max } .
$$

Now, we apply backward rotation $N-g_{\max }$ times to time labeling $T_{1}$, and get a new time labeling

$$
T_{2}=\left[G_{g_{\max }+1}, \ldots, G_{N}, G_{1}, \ldots, G_{g_{\max }}\right] .
$$

If we rename the groups as $T_{2}=\left[\tilde{G}_{1}, \ldots, \tilde{G}_{N}\right]$, using (60) and the fact that (31) is satisfied in time labeling $T_{1}$ for all $g<$ $g_{\min }\left(T_{1}\right)$, it is easy to verify that (31) is satisfied in time labeling $T_{2}$ for all $g<g_{\min }\left(T_{1}\right)+N-g_{\max }\left(T_{1}\right)$, i.e.,

$$
\sum_{j=1}^{g} P_{\tilde{G}_{j}} \geq \frac{g \sum_{k=1}^{K} P_{k}}{N}, \quad \forall g<g_{\min }\left(T_{1}\right)+N-g_{\max }\left(T_{1}\right) \text {. }
$$

In other words, we constructed a new time labeling $T_{2}$, such that

$$
g_{\min }\left(T_{2}\right) \geq g_{\min }\left(T_{1}\right)+N-g_{\max }\left(T_{1}\right)>g_{\min }\left(T_{1}\right) .
$$

Consequently, $\hat{T}$ can be found by carrying out such constructions iteratively.

\section{APPENDIX E \\ PROOF OF THEOREM 5}

Proof: It is easy to verify that if conditions (26) and (27) are satisfied for one time labeling, they are satisfied for all other equivalent time labelings as well. Hence, we focus only on time labeling $\hat{T}$.

Consider the component $[U]_{i m}$ located on the $i$ th row and the $m$ th column. Suppose user $m$ belongs to group $G_{j}$, and assume that $j>i$. Define $\tilde{\boldsymbol{U}}_{1}=\boldsymbol{U}_{i} \ldots \boldsymbol{U}_{1}$ and $\tilde{\boldsymbol{U}}_{2}=\boldsymbol{U}_{N} \ldots \boldsymbol{U}_{i+1}$. We can rewrite matrix $\boldsymbol{U}$ as

$$
\boldsymbol{U}=\boldsymbol{U}_{N} \ldots \boldsymbol{U}_{i+1} \boldsymbol{U}_{i} \ldots \boldsymbol{U}_{1}=\tilde{\boldsymbol{U}}_{2} \tilde{\boldsymbol{U}}_{1}
$$

By following the construction method in Section IV of [3], we can see that $\tilde{U}_{2}$ can be partitioned into

$$
\tilde{\boldsymbol{U}}_{2}=\left[\begin{array}{cc}
\boldsymbol{I}_{\boldsymbol{i}} & \mathbf{0} \\
\mathbf{0} & \#
\end{array}\right]
$$

where the upper-left block is an identity matrix of dimension $i \times i$, and the upper-right block is a zero matrix of dimension $i \times(K-i)$. We are not interested in the entries of the lower-right block, marked with \#. Consequently, $m \in G_{j}$ and $j>i$ gives

$$
[\boldsymbol{U}]_{i m}=\left[\tilde{\boldsymbol{U}}_{2} \tilde{\boldsymbol{U}}_{1}\right]_{i m}=\left[\tilde{\boldsymbol{U}}_{1}\right]_{i m} .
$$

However, according to the construction method, it can also be seen that $\tilde{\boldsymbol{U}}_{1}$ can be partitioned into

$$
\tilde{\boldsymbol{U}}_{1}=\left[\begin{array}{cc}
\# & \mathbf{0} \\
\mathbf{0} & \boldsymbol{I}_{\sum_{n>i}\left|G_{n}\right|}
\end{array}\right]
$$


where the bottom-right block is an identity matrix of dimension

$$
\sum_{n>i}\left|G_{n}\right| \times \sum_{n>i}\left|G_{n}\right|
$$

and the upper-right block is a zero matrix of dimension

$$
\sum_{n \leq i}\left|G_{n}\right| \times \sum_{n>i}\left|G_{n}\right|
$$

Due to the fact that $m \in G_{j}$ and $j>i$, we get $\left[\tilde{U}_{1}\right]_{i m}=0$. Hence,

$$
[U]_{i m}=0, \quad \text { if } m \in G_{j} \text { and } j>i .
$$

From the definition of matrix $\tilde{\boldsymbol{S}}$, it is easy to verify that $\tilde{\boldsymbol{s}}_{k}^{R}=\mathbf{0}$ is satisfied for all $k$. Therefore, (26) holds. Hence, (27) follows straightforwardly from the fact that

$$
\tilde{\boldsymbol{S}} \operatorname{diag}\left(P_{1}, \ldots, P_{K}\right) \tilde{\boldsymbol{S}}^{T}=\frac{\sum_{k=1}^{K} P_{k}}{N} \boldsymbol{I}_{N}
$$

\section{REFERENCES}

[1] S. Verdú, Multiuser Detection. New York: Cambridge Univ. Press, 1998.

[2] M. Rupf and J. Massey, "Optimum sequence multisets for synchronous code-division multiple-access channels," IEEE Trans. Inf. Theory, vol. 40, no. 4, pp. 1261-1266, Jul. 1994.

[3] P. Viswanath and V. Anantharam, "Optimum sequences and sum capacity of synchronous CDMA systems," IEEE Trans. Inf. Theory, vol. 45, no. 6, pp. 1984-1991, Sep. 1999.

[4] S. Ulukus and R. Yates, "Iterative construction of optimum signature sequence sets in synchronous CDMA systems," IEEE Trans. Inf. Theory, vol. 47, no. 5, pp. 1989-1998, Jul. 2001.
[5] P. Viswanath and V. Anantharam, "Optimum sequences for CDMA under colored noise: A Schur-saddle function property," IEEE Trans. Inf. Theory, vol. 48, no. 6, pp. 1295-1318, Jun. 2002.

[6] S. Ulukus and R. Yates, "User capacity of asynchronous CDMA systems with matched filter receivers and optimum signature sequences," IEEE Trans. Inf. Theory, vol. 50, no. 5, pp. 903-909, May 2004.

[7] S. Stanczak and H. Boche, "Optimal signature sequences for asynchronous CDMA systems with fixed signal delays," in Proc. 5th Int. Symp. Wireless Personal Multimedia Communications, Honolulu, HI, Oct. 2002, pp. 27-30.

[8] S. Stanczak, "On sequence sets for symbol-asynchronous CDMA channels with fixed time offsets," in Proc. 37th Annu. Conf. Information Science and Systems, Baltimore, MD, Mar. 2003.

[9] H. Boche and S. Stanczak, "Iterative algorithm for finding optimal resource allocations in symbol-asynchronous CDMA channels with different SIR requirements," in Proc. 36th Asilomar Conf. Signals, Systems and Computers, Pacific Grove, CA, Nov. 2002, pp. 1909-1913.

[10] G. Karystinos and D. Pados, "New bounds on the total squared correlation and optimum design of DS-CDMA binary signature sets," IEEE Trans. Commun., vol. 51, no. 1, pp. 48-51, Jan. 2003.

[11] S. Verdú, "Capacity region of Gaussian CDMA channels: The symbolsynchronous case," in Proc. 24th Allerton Conf. Communication, Control and Computing, Monticello, IL, Oct. 1986, pp. 1025-1034.

[12] - "The capacity region of the symbol-asynchronous Gaussian multiple-access channel," IEEE Trans. Inf. Theory, vol. 35, no. 4, pp. 733-751, Jul. 1989.

[13] R. Gray, "On the asymptotic eigenvalue distribution of Toeplitz matrices," IEEE Trans. Inf. Theory, vol. IT-18, no. 6, pp. 725-730, Nov. 1972.

[14] A. Viterbi and J. Omura, Principles of Digital Communication and Coding. New York: McGraw-Hill, 1979.

[15] R. Bellman, Introduction to Matrix Analysis. New York: McGrawHill, 1960.

[16] W. Yu, W. Rhee, S. Boyd, and J. Cioffi, "Iterative water-filling for Gaussian vector multiple-access channels," IEEE Trans. Inf. Theory, vol. 50, no. 1, pp. 145-152, Jan. 2004.

[17] J. Luo, K. Pattipati, P. Willett, and F. Hasegawa, "Optimal user ordering and time labeling for decision feedback detection in asynchronous CDMA," IEEE Trans. Commun., vol. 51, no. 11, pp. 1754-1757, Nov. 2003.

[18] A. Marshall and I. Olkin, Inequalities: Theory of Majorization and Its Applications. New York: Academic, 1979. 